\title{
Application of Perception Theory in Hotel Interior Design
}

\author{
Hui Yu, Ge Bai, Liang Wu \\ School of Architecture and Fine Art, Dalian University of Technology, Dalian, China \\ Email: 517964304@qq.com
}

How to cite this paper: $\mathrm{Yu}, \mathrm{H} ., \mathrm{Bai}, \mathrm{G}$. and Wu, L. (2018) Application of Perception Theory in Hotel Interior Design. Open Journal of Applied Sciences, 8, 285-295. https://doi.org/10.4236/ojapps.2018.87021

Received: May 25, 2018

Accepted: July 21, 2018

Published: July 24, 2018

Copyright $\odot 2018$ by authors and Scientific Research Publishing Inc. This work is licensed under the Creative Commons Attribution International License (CC BY 4.0).

http://creativecommons.org/licenses/by/4.0/

\begin{abstract}
The purpose of this article is to explore the application of perception theory in hotel interior design. Through the analysis of the theory of perception, the article sums up its connection with the interior design of Modern Hotel, and sums up the hotel interior design method under the influence of three factors of visual perception, space-time perception, logical perception so as to improve the level of humanization. Finally it will provide a basis for hotel interior design in the future.
\end{abstract}

\section{Keywords}

Perception, Hotel, Interior Design, Application

\section{Introduction}

In order to meet the needs of business and tourism arising from economic development, Hotel industry has developed rapidly in recent years. From the tourism data survey of the National Tourism Administration, the hotel industry has entered a golden age of rapid development. With the rapid growth of China's tourism industry, and more frequent business activities in China, the hotel also showed an explosive growth in 2016 as lower travel industry. However, with the improvement of people's living standards and the change of values, the simple provision of accommodation cannot meet the needs of the modern population. The theme hotel with regional culture, folk customs and urban memory is very popular. The interior design of Modern Hotel should not only meet the needs of the hotel itself and development, but also let the design reflect the characteristics of the hotel so as to attract customers to come. Humanization is the soul of hotel design, which puts forward higher requirements for designers. They should take into account the scale, scale, streamline and organization while ensuring the 
comfort of guest rooms and public spaces. Therefore, the designer should start with the human perception, and research the comprehensive experience and emotional input in the hotel environment space, in order to create a humanized hotel room space.

\section{Perception Theory and Hotel Interior Space Design}

\subsection{An Overview of the Theory of Perception}

Perception is the core content of the relationship between human and space. It is a process based on the feeling which is the further organization, recognition and interpretation of sensory information, thus helping people to express and understand the environment. Sensation refers to the active or passive reception and response of human organs to sounds, colors, odors, temperatures, etc. in the environment. Perception adds time dimension, thinking and memory subjective factors on the basis of sensory basis, and there are differences in personality and commonality because of the different background of personal living environment [1]. Therefore, perception is not only the sum of the simple sense information of the environment, such as vision, hearing and touch, but also a comprehensive experience, understanding and emotional input to environmental information. James Gibson, an American cognitive psychologist, thinks that perception is an active pursuit mechanism, not just a passive receiver.

\subsection{The Relationship between Perception Theory and Hotel Interior Design}

A successful hotel design should make use of its own geographical environment, space and regional cultural themes, and explore the material, surface layer and technology in depth that affect the comfort of the people, and make the customers unconsciously intoxicated in a micro and soft way. According to the theory of environmental availability proposed by James Gibson in 1970s [2]. The spatial layout, interface features, materials, components, modeling, furniture, color, ornaments, and lighting are the elements of the hotel's interior design, which can be used to stimulate the active interaction of people and environment so as to find the availability in the environment through perception. For example, when the environment provides customers useful elements, and thereby generates behavior, guides them in the process of perceiving, listening, sniffing, tasting, and touching in the hotel space, and thus stimulates new possibilities. Paying attention to the application of perception theory in hotel interior design is an important procedure for building modern humanized hotel space.

At present, the research of hotel interior design focuses on the orientation of the theme, the inheritance of regional culture, the integration of market economy and the humanized design care, but pay less attention on the space environment design based on the perception of human psychological needs. A study of regional cultural theme hotel interior design based on customer perceived value, from the perspective of customer perception, which has summarized the rela- 
tionship with customer theme atmosphere experience, customer perceived value and customer behavior through a survey of regional cultural theme hotels in Suzhou [3]. Research on interior design style of architectural space based on user perception through different cases of 18 participants in the indoor design style and elements of the perception experiment, summed up the elements of the interior design which influences users' perception [4]. All of these are instructive to the writing of this paper.

\section{Application of Perception Theory in Hotel Interior Design}

The human experience indoor space through three kinds of perceptual levels. The first is visual perception which guides people 1 experience the environment directly, second is space-time perception based on the dynamic experience in the environment, and the third is the logical perception generated by the sports experience in the environment. This paper discusses the application of three kinds of perception in the hotel interior space to guide the humanized interior design method.

\subsection{Visual Perception-Coincidentally Borrowed}

Vision is the direct way to perceive space. People receive information selectively by watching, and feedback information to the brain. The brain produces different psychological responses by judging information, processing and cognition, which is the formation process of visual perception [5]. The difference between it and vision is that vision is a simple presentation of things, and visual perception is the psychological control and grasp of the elements of shape, color, material, and light in space. The designer use the elements such as component modeling, decoration, material and color, which can directly cause visual stimulation in interior design of the hotel, which can directly cause visual stimulation and let customers to explore the space, only in this way, can it infinites expansion of the limited space, and improves the aesthetic and visual feelings of the space. Due to the pursuit of customer attendance and economic benefits, the interior space of Modern Hotel has created many negative spaces. Using local conditions and landscape techniques can join space and function well, with the increase of narrative, the space is extended, and finally the spiritual resonance of the main body can be reached.

Using borrow and delusion as means of enriching indoor visual perception to enhance visual perception. The room interior design of the Lucerne Hotel, designed by Jean Nouvel, pays attention to the use of the roof interface. Its painting inspiration comes from the Renaissance, forms a strong contrast with its elevation, and creates a mysterious and romantic atmosphere for the customers combine the light effect at night (Figure 1). It can also guide the refraction and reflection of light through a series of devices to emphasize the subordination of some function. The Lucerne restaurant is located in the underground space. The lighting is very unfavorable. The interior walls of the building are leaning forward 


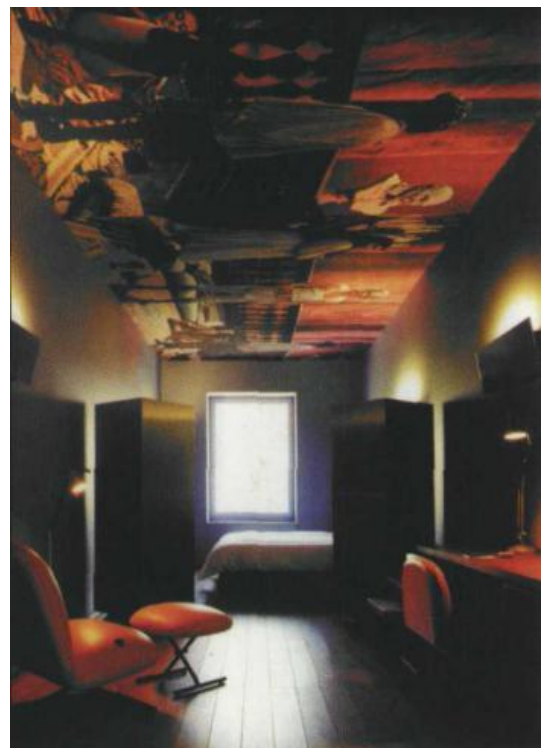

Figure 1. Lucerne hotel room ceiling.

to place glass mirrors with a certain refraction angle. Jean Nouvel uses the angle between the glass mirror, which makes the indoor space abstract and deformed, subverts the normal visual feelings of the people, thus making the space interesting and fascinating. In the Wuyin hotel in Xiamen, China, the refraction glass mirror beside the shared hall will be used to create the borrowing space with the help of partition and natural light. It creates unique light and shadow effects through different angles of light, enrich people's visual perception experience (Figure 2). The third way to enhance visual perception is to break the visual balance by increasing the contrast of spatial levels, emphasizing the special function of one space. For example, the reception hall of citizen $M$ hotel in Paris, a group of visual devices called "refleting holons" is set up to increase the perceived integrity by limiting the area and layer height which can help establish the overall design tone of the hotel (Figure 3). The theme hotel, six Xifeng Road, Baoji, China has created a dramatic stage space. The "bamboo altar" in the central area, the falling red lantern grid, the continuous "immortals map" in the air, and the sinking display platform give the feelings of the public space inside and outside, high and low, far and close to different levels, which have a great visual impact (Figure 4).

Coincidentally borrowed in hotel interior design can stimulate the human visual perception function, it sets up a series of guide devices and increase the space level contrast and other techniques to complete the extension and expansion of space through the rich interface style, but also can get some special space effect, point out the main problem of space environment.

\subsection{Space-Time Perception-Dynamic Experience}

Steven Hall pointed out that changing time, light and sight are the foundation of human perception. The concept of "time" derives from the French philosopher 


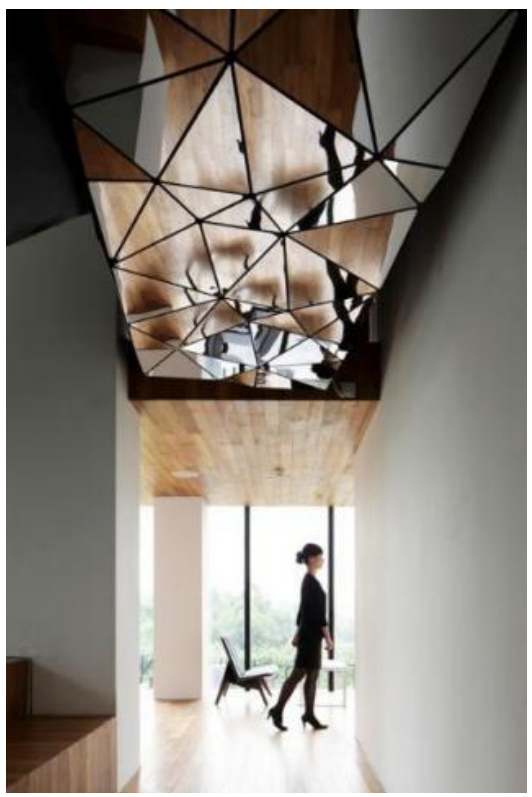

Figure 2. Wuyin Hotel corridor.

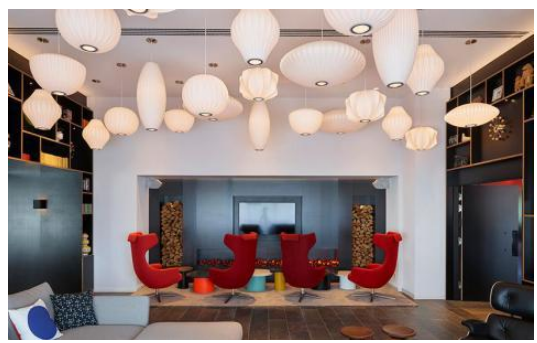

Figure 3. Refleting holons device.

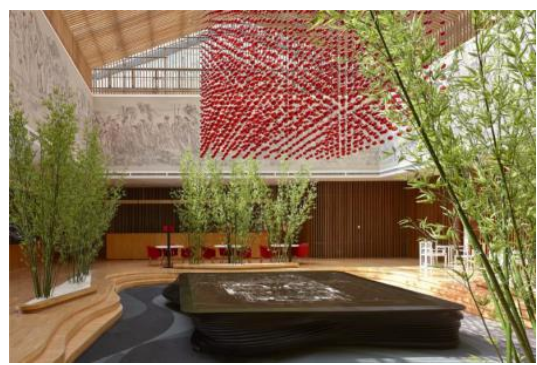

Figure 4. Stage public space.

Bergson, who believes that "the time of life is the real time", space is the "mixture of homogeneous time" [6]. In the indoor space, people know the environment through the change of time and position. In the indoor space, people know the environment through the change of time and position. The concept of "space-time" comes from the integration of time and space, and time is embodied through the movement of space. Space-time perception is different from spatial perception. It is dynamic and continuous, giving users the initiative to explore the environment. In the interior design of the hotel, it breaks through the limit of space scale, and uses human space-time perception to grasp the in- 
teraction of space material, shape, sequence and human, so that space can be used as a continuous experience. The interior design of Modern Hotel has paid the great attention to space experience and feeling, but the stratification and fragmentation is seriously, and there is a phenomenon that the functional cohesion of each part is stiff. If the interaction of man and space can be combined, designers should add dynamic experience to design, and the narrative flow space will be create, so that the whole environment will be enhance.

There are three ways to enhance hotel interior space-time perception. First, we can choose materials with local characteristics as hotel interior decoration materials. In the interior design of Taichung red dot Culture\& Tourism Hotel, designers combine different materials with time and space as the major concept of interior design in subtle ways. For example, a series of materials with time memory, such as firebrick, baked brick and pebble, are used as decorative materials. Furniture also used the old elements in order to achieve a sustainable dialogue between interior decoration and environment, so that completed the continuation of memories in this area (Figures 5-7). Second, designers can try to

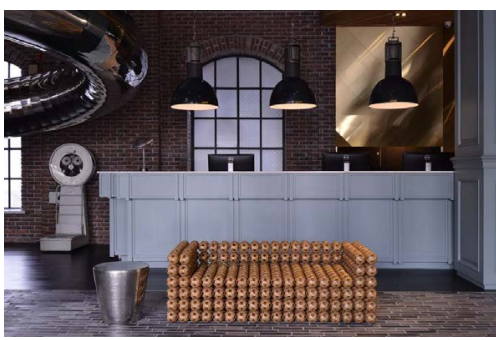

Figure 5. Changes in indoor materials of Taichung red dot Culture \& Tourism Hotel.

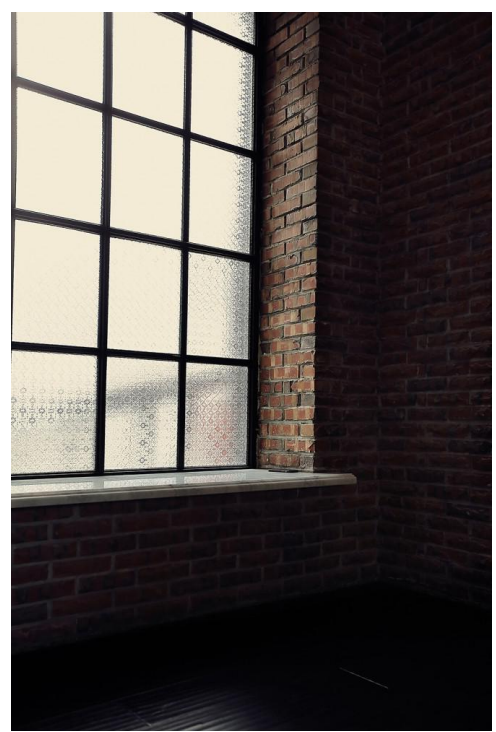

Figure 6. Changes in indoor materials of Taichung red dot Culture \& Tourism Hotel. 


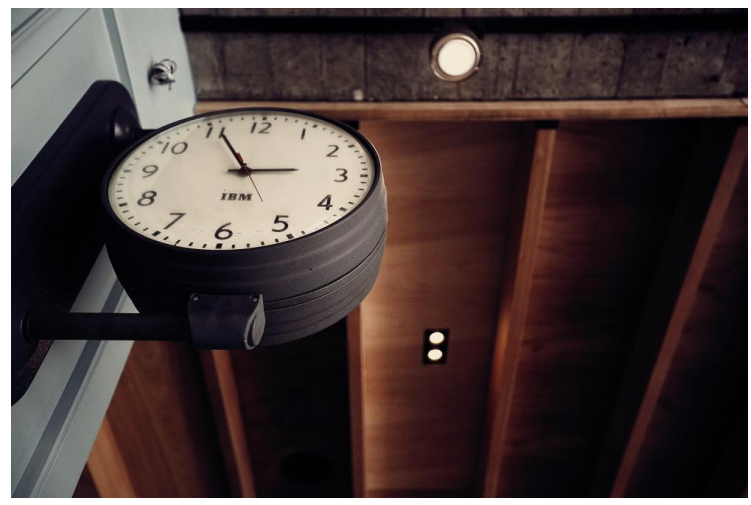

Figure 7. Changes in indoor materials of Taichung Red Dot Culture \& Tourism Hotel.

create "linear" space, for example, taking use of corridors, traffic space, connecting the intersection of the different room and giving space mobility. The landmark stainless steel spiral slide of the public lobby of the hotel is a vertical space component connected to one layer and two layers, making use of the meaning of time and space tunnel to let people feel the controllability of time (Figure 8). Stephen Wooden, the designer of the slide, points out that "slide is not for pleasure, but the symbol of the time and space". Third, in the creation of space sequence, we can use the continuous ceiling, or increase a sense of order with the contrast of high and low, dark, straight and simple, in order to optimize the perception and experience of indoor space. The design of the lobby of Novotel Century Hotel in Hong Kong is decorated with neutral colors, with the orderly arrangement of moss green and golden wood patterns, giving strong guidance to the space. The intention of the arc background also fits well with the dynamic and flexible design concept (Figure 9). Interior design of Beijing Tong Ying Central Continental Hotel spa area takes note of the simultaneous consideration of ceiling and vertical interface. The contrast between "light" and "dark", "wide" and "narrow", "real" and "virtual" strengthen the spatial attributes, give the space "moving" trend, and virtually enrich people's space-time perception (Figure 10).

People's feeling in the hotel interior space is enhanced by delaying time in perceiving rhythm. The change of material, the contrast of the shape component and the construction of the sequence space all expand and extend the human consciousness, give the intention of the space experience and perception, and stimulate the people's pursuit of the quality of the better life.

\subsection{Logical Perception-Associative Inference}

The logical thinking is formed in the condensed stage. It is the deepest stage of the spatial emotion experience. And it is a complement and deep reaction process of people to perceive things. It is a purely subjective thinking process [7]. Gestalt psychology's Gestalt theory points out that the blank design, the virtual reality and the minimalism in the interior design will exert the space experience 


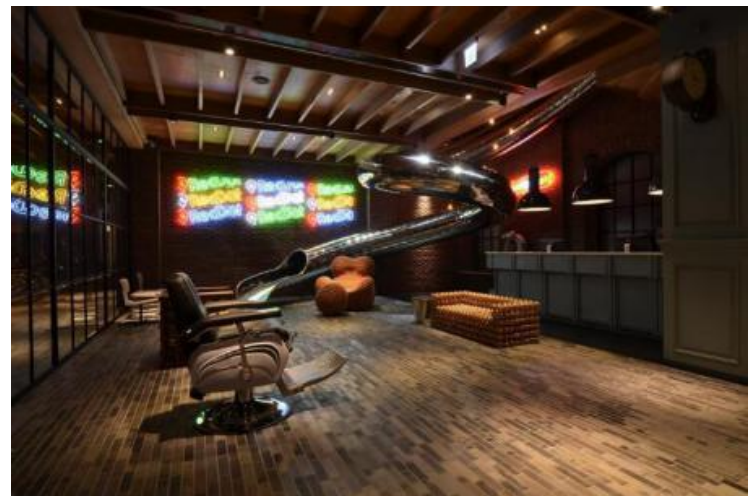

Figure 8. The slide of the space.

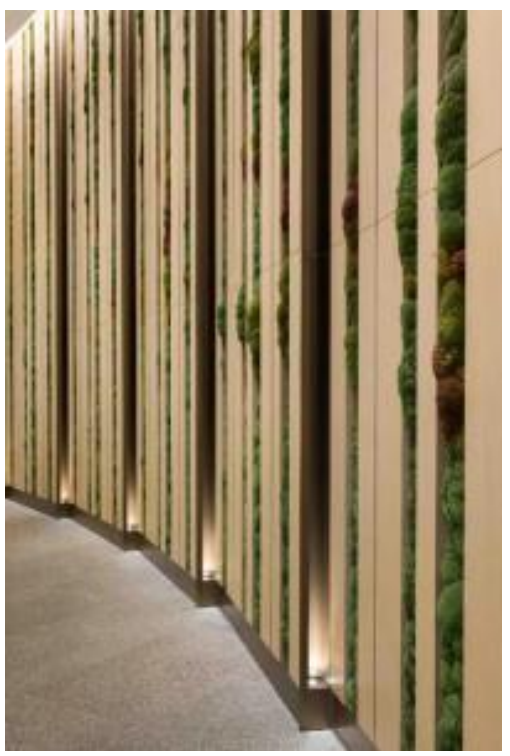

Figure 9. Space sequence.

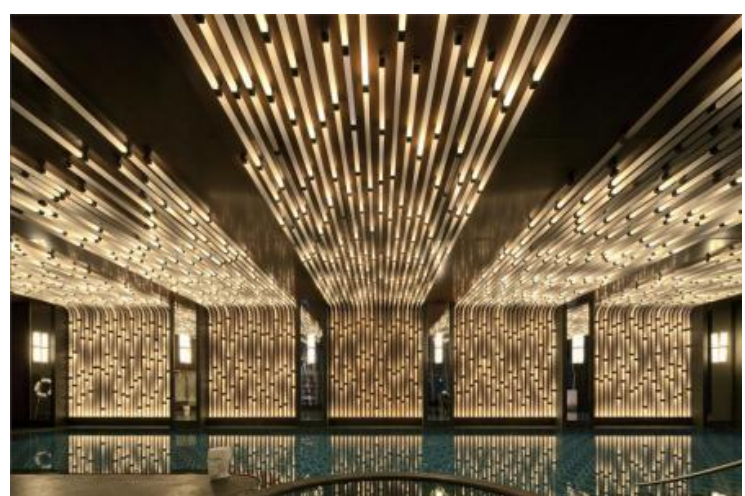

Figure 10. Spa area ceiling.

to the extreme. Artistic conception comes from human reflection experience. This reflection has a deep influence on the reconstruction of human consciousness and spatial cognition [8]. Logical perception is the evaluation of the environment after visual perception and space-time perception. It is a perceptual ex- 
pression transformed through the user's experience, and is the subjective self feedback of the user. In the interior design of the hotel, the contrast of light and material and the creation of new technology are helpful to guide people to think and associate the space environment. It is an important means to create the spiritual space. With the development of the technology, the interior design style of the hotel tends to be scientific and personalized. How to take account of the spiritual and cultural implants under the development of the new technology is an important proposition of the Modern Hotel design.

The method of enhancing the logical perception of indoor space in the hotel can make use of clear spatial composition and simple furniture streamline to divert the mind of people, so that they can talk to space. Cloud water Pavilion in Thousand Islet Lake creates a simple living space by clean, concise and sophisticated design methods. The unsupported floating bar contrasts sharply with the white background wall, which causes people's suspicion and imagination. However, with the change and experience of space, the psychological balance between customers in transformation and comparison has been established, and doubts have been answered. Infinitely space enlarges the measurement of people about thinking and feeling environment to another extent, triggering people's process from "association" to "inference" (Figure 11 and Figure 12). Secondly, designers can add lighting, temperature independent selection, control system to provide users with better quality in the design of guest room, and use the thinking to control the concept of humanized service. The intelligent booking system of Hong Kong Sleeping hotel is a model of Modern Hotel design, guests can choose the thickness of bed mattress, pillow size and quilt thickness according to their preferences by online booking system. At the same time, every dormant room has an adjustable indoor temperature and an indirect lighting device, which can make the indoor temperature and light controllable, so that to make the sleeping room more suitable for users' needs (Figure 13).

Logical perception aims to use process of "reasoning", "association" and "utilization" into the interior design of the hotel, which is helpful to the reflection of the objective things. A good hotel space will reappear in the consciousness of the

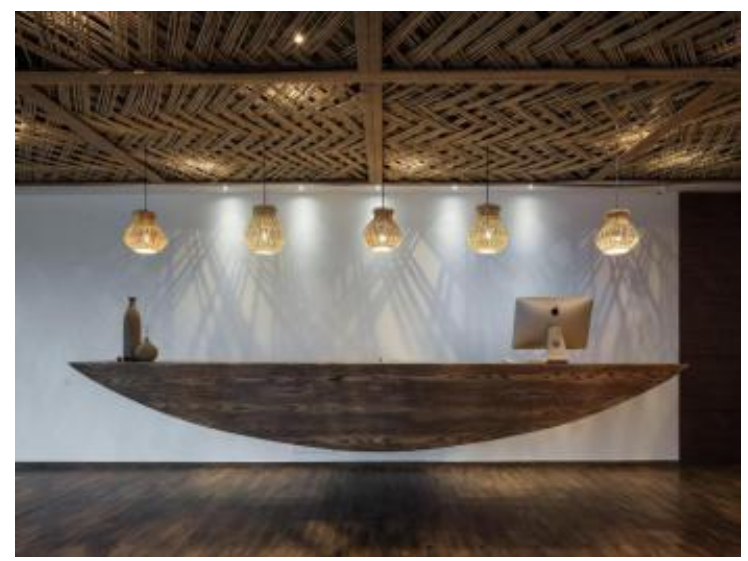

Figure 11. A floating bar. 


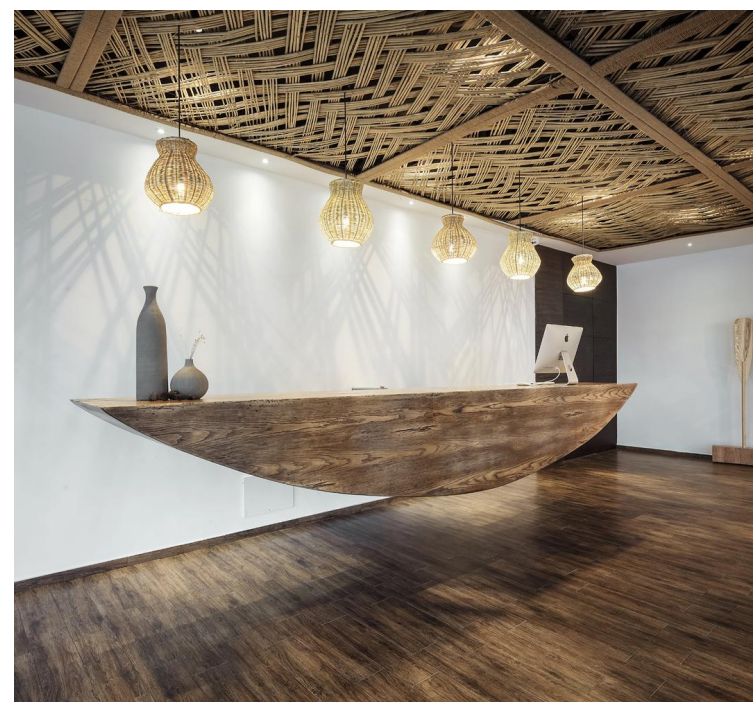

Figure 12. A floating bar.

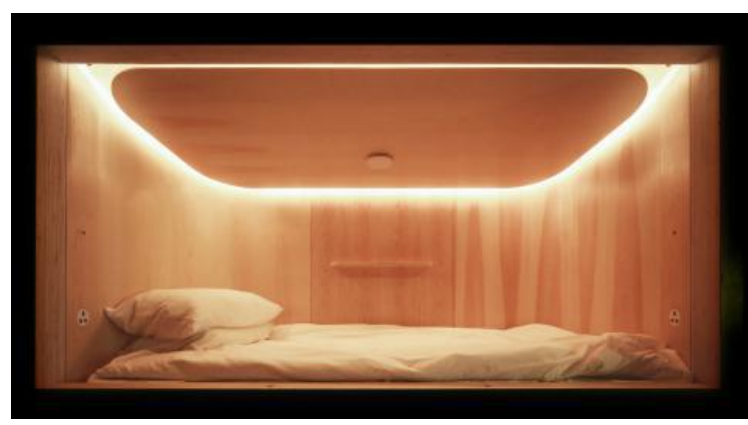

Figure 13. Sleeping room.

human brain under the stimulation of a certain external environment, making it a place to retain memories.

\section{Conclusion}

Through the in-depth analysis of visual perception, space-time perception, logical perception and the case analysis of its application in hotel interior design, we can find that the three ways of interweaving and complementing each other are the effective ways to optimize the indoor space of Modern Hotel. Visual coincidentally borrowed increases the creativity of interior design by breaking through the limitations of interior space. The dynamic experience of space-time strengthens the interaction between human beings and substance through the "four dimensions" of time. Logical association inference gives users initiative, completes the exchange of thinking between objects, and enriches the humanization experience of hotel interior space. Applying the perception theory to the hotel interior design can enhance the humanization level of the hotel, meet the diversified needs of customers, and get the customers' affirmation. It is the soft power of the hotel development, will also guide the sustainable development of the hotel, and brings more economic and social benefits for the hotel, so this study has 
a good practical significance. Only in this way, can we put the design method that accords with people's perception rules into practice, and on the other hand, the design concept of hotel interior space is increasingly strengthened.

\section{References}

[1] Victor, S. (2015) City Perception-The Secret Dimension in Urban Places. China Architecture \& Building Press, Beijing.

[2] Gibson, J.J. (1996) The Ecological Approach to Visual Perception. Lawrence Erlbaum Associates, Hillsadle, New Jersey.

[3] Gu, Q.C. (2016) A Study of Regional Cultural Theme Hotel Interior Design Based on Customer Perceived Value. M.S. Thesis, Suzhou University, Suzhou.

[4] Wang, D.D., Qin, H. and Xing, H.N. (2017) Research on Interior Design Style of Architectural Space Based on User Perception. Industrial Building, Supplement II, 8.

[5] Hu, J. (2011) A Study on the Visual Perception of Interior Space and Form. M.S. Thesis, Southwest Jiaotong University, Chengdu.

[6] Wang, B. (2013) The Primary Study of Architectural Space Based on Multi-Level Perception. M.S. Thesis, Heifei University of Technology, Heifei.

[7] Huang, H.Q. (2014) Experience Space-The Research on Architecture Space Experience Based on Architecture Phenomenology. M.S. Thesis, Shandong Jianzhu University, Jinan.

[8] Sun, W. (2015) Study on the Narrative Design Techniques of Indoor Space. The study of Art Education, 17, 86-87. 